\title{
Derechos humanos, guerra, posguerra y memoria en El Salvador: Sobre la vida y la obra académica de Ralph Sprenkels
}

\author{
Human Rights, War, Postwar and Memory \\ in El Salvador: About Life and Academic \\ Work of Ralph Sprenkels
}

\section{Direitos humanos, guerra, pós-guerra e memória em El Salvador: sobre a vida e a obra acadêmica de Ralph Sprenkels}

\author{
Lidice Michelle Melara Minero* \\ Mauricio Menjívar Ochoa**
}

\begin{abstract}
Resumen: El antropólogo e historiador de origen holandés Ralph Sprenkels murió inesperadamente en septiembre de 2019. Sprenkels dedicó la mayor parte de su investigación a entender y explicar el conflicto armado y la posguerra en El Salvador. El presente artículo es un recorrido sobre cómo Sprenkels se vinculó a dicho país y el impacto que tuvo en su vida. Sprenkels decidió involucrarse en el trabajo a favor de los derechos humanos y, posteriormente, se dedicó a la investigación de la polarización política en la posguerra, al estudio de la memoria y la justicia transicional, así como a comprender la dinámica de la posguerra de uno de los movimientos revolucionarios más importantes de Latinoamérica: el FMLN. En este sentido, el artículo realiza un recuento general de su obra, a partir
\end{abstract}

Fecha de recepción: 20/06/2020 - Fecha de aceptación: 13/07/2020

* Salvadoreña-holandesa. Máster en Ciencia Política por la Universidad José Simeón Cañas (UCA), San Salvador, El Salvador. Investigadora independiente y miembro del Grupo de Trabajo O ITSMO Centroamérica del Consejo Latinoamericano de Ciencias Sociales (CLACSO).

Correo electrónico: melaraminero@yahoo.es. ORCID: https://orcid.org/0000-0001-6224-1414.

** Costarricense-salvadoreño. Doctor en Historia por la Universidad de Costa Rica (UCR), San José, Costa Rica. Docente e investigador en la Escuela de Estudios Generales de la Universidad de Costa Rica (UCR), San José, Costa Rica. Correo electrónico: mauricio.menjivar@ucr.ac.cr.

ORCID: https://orcid.org/0000-0003-1199-8091. Mi participación en este artículo se inscribe en el proyecto de investigación «Anti-sujetos masculinos: Militares, guerra civil y crueldad en El Salvador, 1981$1983 »$, con vigencia durante los años 2020 a 2022 (N. ${ }^{\circ}$ C0107). 
de lo cual concluye que el trabajo académico de Sprenkels constituye un aporte significativo para la comprensión de la realidad salvadoreña, y que realiza contribuciones teóricas relevantes para nuevas investigaciones.

Palabras claves: El Salvador; (pos)conflicto; guerra civil; Derechos Humanos; historia; Ralph Sprenkels; memoria.

\begin{abstract}
Dutch born anthropologist and historian Ralph Sprenkels, died unexpectedly in September 2019. Sprenkels devoted most of his research to understanding and explaining the conflict and post-war in El Salvador. This article describes the journey of how Sprenkels became linked to El Salvador and the impact this had on his life. He became a human rights activist, and later, dedicating himself to the investigation of postwar political polarization, to the study of memory and transitional justice, as well as one of the most important revolutionary movements in Latin America: the FMLN. In this sense, the article provides a general account of his work, which concludes that Sprenkel's academic work constitutes a significant contribution to the understanding of Salvadorian reality, making significant theoretical contributions to new research.
\end{abstract}

Keywords: (Post)conflict; El Salvador; civil war; Human Rights; history; Ralph Sprenkels; memory.

Resumo: O antropólogo e historiador holandês Ralph Sprenkels faleceu inesperadamente em setembro de 2019. Sprenkels dedicou a maior parte da sua pesquisa para entender e explicar o conflito armado e o período pós-guerra em El Salvador. Este artigo é um percurso sobre como Sprenkels está ligado a este país e o impacto que isso teve em sua vida. Sprenkels decidiu empreender um trabalho de direitos humanos e, subsequentemente, dedicou-se a pesquisar a polarização política do pós-guerra, estudar a memória e a justiça de transição, bem como compreender a dinâmica do pós-guerra de um dos movimentos revolucionários mais importantes da América Latina: o FMLN. Nesse sentido, o artigo faz um traço geral de sua obra, a partir do qual conclui que o trabalho acadêmico de Sprenkels constitui uma contribuição significativa para a compreensão da realidade salvadorenha, e que ele dá contribuições teóricas relevantes para novas pesquisas.

Palavras chaves: El Salvador; (pós) conflito; guerra civil; Direitos Humanos; história; Ralph Sprenkels; memória. 
Above all, I have been a sentient being, a thinking animal, on this beautiful planet, and that in itself has been an enormous privilege and adventure. Oliver Sacks ${ }^{1}$

\section{Introducción ${ }^{2}$}

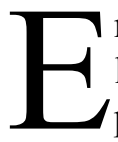

n la oficina de su casa, Ralph Sprenkels tenía la cita de Oliver Sacks con la que introducimos este artículo. Estaba impresa y pegada en una de las paredes al lado de una pañoleta negra del Primer Encuentro de Exguerrilleros(as) en el Arrozal, Chalatenango (1998). Estos dos elementos, un símbolo al lado del otro, no los colocó Ralph por casualidad. La historia reciente de El Salvador y su continua interpelación a esta fueron parte esencial de su vida y marcaron su trabajo intelectual. La cita de Sacks describe cómo Ralph se veía y era en este mundo, un ser humano en continuo aprendizaje, haciéndose y haciendo preguntas sobre el conocimiento y, particularmente, sobre el proceso político salvadoreño. Ralph se identificaba con las palabras de Sacks porque daban cuenta de su propio sentimiento de afecto, siempre profundo e intenso, por la vida, el privilegio que sentía de poder hacer las cosas del modo que las hacía. Para Ralph, el paso transitorio por este mundo era una aventura que había que inventar continuamente dándole significado.

Tomando como referencia la cita de Sacks, en el presente trabajo, quisiéramos mostrar cómo el intelectual holandés Ralph Sprenkels -9 de marzo de 1969-14 de septiembre de 2019- se embarcó en la aventura del conocimiento y se volcó casi toda su vida a participar, reflexionar e investigar sobre el (pos) conflicto salvadoreño. En las tres primeras partes, hacemos un recorrido por el proceso de búsqueda vital y el involucramiento de Ralph en la problemática de El Salvador de la posguerra, país en el que jugó un papel de primer orden en pro de los derechos humanos. A partir de la cuarta parte, procuramos realizar una aproximación a su obra intelectual publicada en español y en inglés para, finalmente, señalar su aporte en el juicio por la masacre de El Mozote, a inicios de la década de 1980.

2 Los textos originarios en inglés han sido traducidos por Lidice Michelle Melara Minero, y revisados por Gabriela Carrión quien es argentina, Licenciada en Letras Modernas y Técnica en Corrección Literaria por la Universidad Nacional de Córdoba (UNC), Argentina. Actualmente se desempeña como correctora literaria independiente. Correo electrónico: gabrielacarrion@hotmail.com. A Gabriela, le agradecemos la lectura de este artículo y sus comentarios. 


\section{Cruzando el charco ${ }^{3}$}

Ralph Sprenkels acababa de terminar su primer año de filosofía en la Universidad de Ámsterdam en los Países Bajos cuando decidió hacer un giro radical en su vida: dejó de estudiar y armó un viaje a los Estados Unidos. Su elección era un camino incierto, lo sabía. Era el comienzo de un viaje interesante, de búsquedas y (des)encuentros. Una travesía develadora para un joven de 20 años. Acompañado de Martijn Bijlsman, un amigo de pláticas filosóficas interminables, cruzaron el océano Atlántico y emprendieron el viaje. Ambos llegaron a Michigan, donde los familiares de Ralph los acogieron. En esta casa, un mundo nuevo se fue abriendo. Ahí sonaba música folclórica de Cisco Houston, como su famosa canción Deportees, y las voces emblemáticas de Joan Báez y Lead Belly, entre otros. Cantantes norteamericanos que mezclaban los valores musicales con pensamientos políticos. Ralph, un enamorado de la música, sumó inmediatamente a estos cantantes a su lista de canciones preferidas. Además de la música, se apasionó por la literatura y la poesía norteamericana. La cultura, el paisaje y la gente del país del norte se convirtieron en un continuo referente de su vida.

Después de un par de meses en Michigan, Martijn y Ralph decidieron continuar su viaje. Compraron un automóvil, un Volkswagen combi bus, de esos largos de once ventanas y tres puertas. En él viajaban y vivían. No había reglas ni tiempos. En algunos lugares se quedaban días, en otros, meses. Para sobrevivir y poder seguir viajando, Ralph realizaba diversos trabajos: se dedicó a cortar leña, aprendió el oficio de brocha gorda y pintó casas y trabajó en restaurantes lavando platos, pero lo que más le gustaba era cuando lo contrataban de cocinero: se hizo bueno en la cocina hasta el punto de convertirse en chef de un restaurante.

Juntos recorrieron casi todo el país. Después de un año, Martijn decidió que su viaje había concluido y regresó a los Países Bajos. Para Ralph, la aventura apenas había comenzado. Vendió el automóvil, cruzó la frontera de los Estados Unidos y empezó su viaje por México. En bus conoció varios Estados mexicanos. Y al llegar a Guadalajara, decidió quedarse. Encontró una casa donde vivían varios estudiantes extranjeros. Ahí se debatía sobre la lucha política y social en América Latina. Ralph rápidamente se sintió cómodo en su nueva casa, entre jóvenes estudiantes y activistas. Al poco tiempo, decidió entrar a estudiar historia en la Universidad de Guadalajara.

Estando en esta ciudad, Ralph se involucró en un movimiento de solidaridad con El Salvador. Colaboraba en diferentes actividades: ayudaba en la guardería a cuidar niños refugiados salvadoreños y a veces trabajaba en la pupusería que funcionaba los fines de semana. Ralph participaba activamente en las reuniones del

3 Expresión que se utiliza para decir que se va a cruzar el océano Atlántico. 
movimiento, asistía a las pláticas que se organizaban, iba a los eventos culturales. ${ }^{4}$ En menos de un año, estaba inmerso en el movimiento político y fue adquiriendo cada vez más responsabilidades, a tal punto que, después de un año, dejó sus estudios en la Universidad de Guadalajara y se metió de cabeza en su trabajo.

\section{«El otro lado...mi nuevo mundo» ${ }^{5}$}

Tras la firma de los Acuerdos de Paz en El Salvador (1992), Ralph se trasladó de Guadalajara, México, a San Salvador, El Salvador, y continuó su trabajo con refugiados y comunidades repobladas en una organización no gubernamental. Al poco tiempo de haber llegado, se mudó de la capital a Chalatenango, departamento ubicado al norte del país. En este lugar conoció a Jon Cortina S. J. (1934-2005), quien trabajaba en la región, su experiencia en las comunidades era vasta. Desde 1978 había vivido en el municipio de Aguilares, donde llegó poco después de que mataron a Rutilio Grande García S. J. (1928-1977). Cortina vivió en Aguilares cuando la represión política contra la iglesia, particularmente contra los jesuitas, era intensa. ${ }^{6}$ En 1981 se retiró de Aguilares, y en los siguientes años del conflicto armado acompañó a las comunidades de Chalatenango. ${ }^{7}$ Ralph aprendió del trabajo y la experiencia de Cortina, y con su propia capacidad y habilidad empezó a adentrarse en la vida de las comunidades campesinas; a comprender la tragedia, el dolor, la pobreza, las carencias que la gente vivía; pero también descubrió la solidaridad, la vitalidad, la entereza, la creatividad y la esperanza que la conformaba.

Cuando se nombró la Comisión de la Verdad (CdV) para esclarecer las violaciones a los derechos humanos que se habían cometido durante el conflicto armado (1980-1992), Cortina decidió colaborar y formó un equipo en el cual Ralph y otros más participaron. La primera tarea fue convencer a la $\mathrm{CdV}$ de que llegara a Chalatenango, en vez de que los pobladores fueran a San Salvador a rendir su declaración, tal como dicha comisión esperaba. Según Cortina, los argumentos del equipo eran claros: la gente aún tenía miedo de ir a San Salvador y necesitaba declarar en un lugar donde sintiera seguridad, era una denuncia personal y específica en contra del Estado sobre crímenes que este había cometido. Además, el viaje también requería gastos económicos por parte de los pobladores, quienes no tenían cómo pagarlos. ${ }^{8}$ El gran logro del equipo fue que la $\mathrm{CdV}$ se ubicara en diferentes puntos de Chalatenango para recibir las denuncias de sus pobladores.

4 Ralph Sprenkels, «Revolution and Accommodation. Post-Insurgency in El Salvador» (Tesis de Doctorado, Universidad de Utrecht, Países Bajos, 2014b), 2.

5 Expresión tomada del Diario, no publicado, de Ralph Sprenkels, incluida en sus anotaciones del 6 de julio de 1993.

6 Ralph Sprenkels, ed., Caminar con el pueblo. Entrevista con Jon Cortina (San Salvador, El Salvador: Ediciones Populares, 2009).

7 Ibíd., 26-27.

8 Ibíd., 38-39. 
Previo a la llegada de la $\mathrm{CdV}$, el equipo realizó varias tareas que facilitaron su trabajo y la construcción del relato de los pobladores. Elaboraron un formato para la recolección de datos con la ayuda del Instituto de Derechos Humanos de la Universidad Centroamericana José Simeón Cañas (UCA) con base en las preguntas que solía realizar la $\mathrm{CdV}$. Con este formato, el equipo empezó la labor de visitar a los pobladores en todo Chalatenango. La idea era llenarlo para que la gente lo llevara preparado antes de la entrevista con la CdV. Ralph visitó caserío tras caserío para conversar con las propias víctimas y familiares de las víctimas del reciente conflicto armado que quisieran compartir su historia con él. En el proceso descubrió que la recolección de la información no sería fácil, así lo describe él mismo:

\begin{abstract}
Chalatenango se dividió en dos partes para nosotros: donde fuimos recibidos con los brazos abiertos y la gente hacía cola para hablar con nosotros. Y, donde tuvimos que hacer contactos cuidadosamente y nadie quería ser visto con nosotros. La línea divisoria entre los dos Chalatenangos corría aproximadamente entre lo que estaba en manos del FMLN durante la guerra y lo que era el territorio del ejército. Santa Rita fue uno de esos lugares que llamamos pueblos de miedo, ubicados a pocos kilómetros de la instalación militar más grande de la provincia. Después de muchos intentos, finalmente encontramos un contacto dispuesto a hablar sobre lo que había sucedido durante la guerra. Habíamos decidido que yo iría porque no se me conocía como afiliado al FMLN, y me sentiría más seguro para nuestro contacto, el maestro local. Cuando anuncié mi visita en el portón, miró a su alrededor asustadizo antes de apresurarme a entrar. Luego cerró la puerta y la persiana de la ventana. Me susurró nerviosamente tan bien y tan enojado como su memoria le permitía el informe de una docena de asesinatos y desapariciones que habían tenido lugar en Santa Rita. Hablar con los familiares de las víctimas sería imposible porque estaban paralizadas por el miedo. Muchos de los perpetradores aún vivían en el pueblo. Y aunque como paramilitares estaban desarmados formalmente, continuaron amenazando e intimidando. ${ }^{9}$
\end{abstract}

El equipo visitó docenas de pueblos durante seis meses. Finalmente, presentaron un total de 3118 casos individuales entre asesinatos, desapariciones forzadas y violaciones, y reportaron cerca de 80 masacres que habían ocurrido en Chalatenango entre 1979 y $1992 .{ }^{10}$ Los casos encontrados por el equipo abarcaban un período más amplio que el marco establecido por la CdV. Los datos mostraban que la mayor parte de las violaciones habían sido cometidas por el ejército salvadoreño, las fuerzas de seguridad y las unidades paramilitares. A las fuerzas guerrilleras del Frente Farabundo Martí para la Liberación Nacional (FMLN), se le atribuyeron un número menor de violaciones.

9 Ralph Sprenkels, «Een messcherpe scheidslijn. Chalatenango, El Salvador: Toen en nu», en Overleven Verplicht. 25 jaar na de moord op de Nederlandse jounalisten in El Salvador, uitgegeven door Marc Broere (Amsterdam, Países Bajos: OnzeWereld Media, 2007), 58.

10 Ibíd. 
La mayor parte de denuncias era sobre muertos y desaparecidos. Muchos de los pobladores hablaban de los desaparecidos como si estos estuvieran muertos, pero durante el proceso de recolección de datos, el equipo fue descubriendo que no todas las denuncias de desaparecidos significaban la muerte del familiar. Así comenzaron las preguntas sobre la posibilidad de que algunos estuvieran vivos, particularmente niños y niñas, y si lo estaban, dónde estaban y cómo podían encontrarlos. ${ }^{11}$ Había un caso en particular que el equipo presentó a la CdV, el caso de Magdalena Ramos -Mayda-, quien había perdido a su hijo durante un operativo. Dicho caso indicaba que habían existido prácticas de desaparición forzada y la posibilidad de que el niño aún estuviera con vida. ${ }^{12}$

El trabajo previo del equipo facilitó la recolección de los testimonios y registrar mayor cantidad de casos. $\mathrm{La} \mathrm{CdV}$, que tenía poco tiempo y escasos recursos materiales y humanos, pudo así registrar la información, verificarla y agregar datos que considerara necesarios en el momento en que visitó Chalatenango. En ocasiones, cuando la CdV lo necesitaba, Ralph llevaba a la gente de Chalatenango a San Salvador. En este proceso fue creciendo la relación entre ambos. ${ }^{13}$

Escuchar día tras día el drama que aquellas comunidades habían vivido durante el conflicto tuvo un gran impacto en Ralph y su equipo, a veces tenían pesadillas y no lograban dormir. Cada historia era desgarradora. Ralph se dio cuenta, en poco tiempo, que Chalatenango tenía una historia que contar, ahí había pasado algo intenso, dramático y glorioso a la vez. Ese lugar era testigo de un drama sin precedentes. Tal como él mismo escribe en su último libro: «las montañas de Chalatenango revelaban una geografía muy específica del terror. Cada comunidad tenía sus cuentos de terror de frenesí asesino. Restos no enterrados, esparcidos en laderas desoladas». ${ }^{14}$

En el libro de notas que escribió durante sus años en Chalatenango (19921995), revela su sentimiento en cuanto a la tragedia vivida por esas comunidades: «las carreteras de Chalatenango deberían de estar grabadas con el recuerdo de "un cadáver tras otro", un camino de cadáveres, una línea de cruces, todo el camino hasta Honduras». ${ }^{15}$

11 Sprenkels, Caminar con el pueblo..., 43.

12 Ralph Sprenkels, ed., El día más esperado. Buscando a los niños desaparecidos de El Salvador (San Salvador, El Salvador: Asociación Pro-Búsqueda de Niñas y Niños Desaparecidos/UCA Editores, 2001), 69-82.

13 Sprenkels, Caminar con el pueblo..., 39.

14 Sprenkels. Revolution and Accommodation ..., 7.

15 Ibíd., 7. 


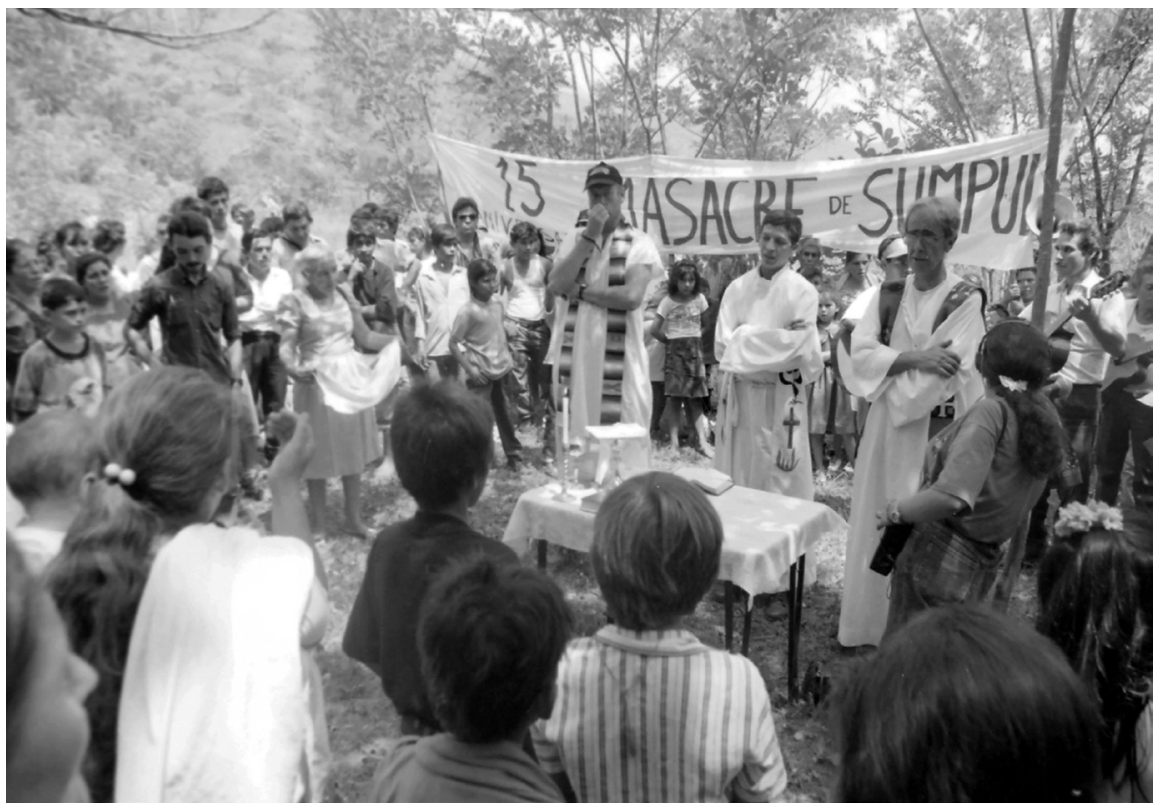

Fuente: Fotografías de Ralph Sprenkels CRalph Sprenkels. Con permiso de Derechos de autor de la familia Sprenkels Melara.

Nota: La fotografía fue tomada durante la conmemoración del 15 aniversario de la masacre de Sumpul en mayo de 1995. Oficiando la misa, a mano derecha, Jon Cortina. La masacre donde murieron aproximadamente 600 campesinos, ocurrió el 14 de mayo de 1980 en el caserío Las Aradas situado a orillas del río Sumpul. Esta fue perpetrada por fuerzas militares salvadoreñas combinadas en colaboración con el ejército hondureño, este último, impedía el paso de los pobladores salvadoreños a través del río.

El equipo entregó la información recopilada a la CdV. Esta incorporó una parte de los datos en el informe De la locura a la esperanza, publicado el 23 de marzo de 1993. Ralph, al igual que mucha gente, esperaba que el informe brindara justicia, reparación y compensación a los familiares con los que habían trabajado. Esta esperanza se vio derrotada cuando, cinco días después de la presentación del informe, la Asamblea Legislativa decretó la Ley de Amnistía. Además de esta desilusión, le sorprendió que en el informe no aparecieran los casos de desaparición forzada de niños y niñas como una práctica sistemática, y que no adquirieran especial atención en un apartado, sino que habían sido sumados a la larga lista de desaparecidos en uno de los anexos.

Ralph y Jon siguieron trabajando en el equipo y formaron un comité de derechos humanos en Chalatenango. Uno de sus principales objetivos era crear una voz de denuncia en el espacio público. Comenzaron difundiendo el informe de la $\mathrm{CdV}$ en el departamento, incluso en las áreas controladas por el ejército salvadoreño y los grupos paramilitares, lo cual aún era riesgoso en los primeros años de paz. Con base en la información que habían recabado durante su trabajo 
con la $\mathrm{CdV}$, decidieron iniciar un proyecto de investigación sobre los casos de desaparición forzada de niños y niñas realizados durante los operativos contrainsurgentes llevados a cabo por el ejército y los grupos paramilitares. El esfuerzo del comité iba paralelo al de los familiares de niñas y niños desaparecidos, quienes se buscaban y organizaban para construir un sentido común.

En 1993, el comité encontró un grupo de 6 niños y niñas en un orfanato de Aldeas Infantiles SOS cerca de la capital. El 16 de enero de 1994 se llevó a cabo el primer reencuentro en Guarjila, Chalatenango; entre ellos estaba el hijo de Mayda. A partir de este encuentro, el comité tuvo «una avalancha de casos de niños desaparecidos» durante la guerra y que posiblemente estaban con vida, viviendo con diferente identidad. ${ }^{16}$ De esta manera, decidieron iniciar una organización de familiares y activistas de derechos humanos llamada Pro-Búsqueda, fundada en agosto de 1994, de la cual Ralph fue cofundador. ${ }^{17}$

\section{La búsqueda de la memoria}

Después de vivir tres años en las comunidades de Chalatenango, Ralph se mudó en 1995 a la capital, donde Pro-Búsqueda ubicó su oficina central. En los siguientes años, se dedicó de lleno a la investigación de niños y niñas desaparecidos durante el conflicto. Junto con el equipo de Pro-Búsqueda, Ralph documentaba de manera meticulosa caso por caso, utilizaba técnicas de investigación como las de los detectives, ayudaba en la construcción de la base de datos de ADN y colaboraba con otras organizaciones de derechos humanos.

Durante la posguerra, el trabajo de Pro-Búsqueda era intenso y no dejó de ser, en ocasiones, peligroso. Ralph acompañó a los familiares a poner las denuncias ante los tribunales de justicia, pero en la mayoría de las ocasiones no tuvieron éxito. También intentó conversar y obtener documentos de la Fuerza Armada, pero el acceso le fue continuamente denegado. Las campañas públicas para encontrar a los niños y niñas desaparecidas creaban tensiones en el escenario político; Pro-Búsqueda fue varias veces amenazada.

Gracias al compromiso, al trabajo arduo y constante, y a la meticulosa labor que hacían Ralph y el equipo de Pro-Búsqueda, se lograron importantes resultados, el más valioso: centenares de reencuentros entre los familiares y los niños y niñas desaparecidos. Se creó una base de datos de niños y niñas desaparecidos bien documentada, denuncias de casos ante los tribunales de justicia, y una organización de derechos humanos capaz de sostenerse y tomar voz pública en la posguerra en una sociedad que aún tiende a olvidar su historia reciente, y donde las violaciones de los derechos humanos durante la guerra aún son negados por sus perpetradores.

La experiencia de investigación sobre niñez desaparecida llevó a Ralph a apoyar el trabajo de la Oficina de Derechos Humanos del Arzobispado de Guatemala (ODHAG). En dicho país, al igual que en El Salvador, la desaparición de 
niños y niñas durante el conflicto armado había estado estrechamente vinculada «con el contexto de las masacres» ${ }^{18}$ llevadas a cabo en las comunidades campesinas. Ralph trabajó de cerca con el equipo de la ODHAG que produjo el informe Hasta encontrarte.

Durante su trabajo en Pro-Búsqueda, Ralph escribió dos libros: El día más esperado e Historias para tener presente, publicados en 2001 y 2002, respectivamente. Ambos abordan, desde diferentes perspectivas, la desaparición forzada de niños y niñas ejecutada por el ejército salvadoreño y los grupos paramilitares durante la guerra. Asimismo, aparecen señalamientos acerca de la compleja relación que se vivía dentro del FMLN, las rivalidades y luchas de poder que en muchas ocasiones llevaron a prácticas de violencia interna en la organización de izquierda, y la utilización de niños y niñas dentro de las casas de seguridad. Mientras trabajaba en la producción de sus libros, Ralph coordinó, junto con otras organizaciones de derechos humanos, la construcción del Monumento a la Memoria y la Verdad, en homenaje a las víctimas civiles, en donde se grabaron más de 25 mil nombres entre desaparecidos y asesinados, ubicado en el parque Cuscatlán.

\section{El viraje a la academia}

Tras casi una década de vivir en El Salvador, Ralph volvió a los Países Bajos. La decisión de mudarse y cruzar nuevamente el charco no fue fácil. El Salvador era el lugar donde se sentía en casa, pero al mismo tiempo, algo había cambiado en el país, en él. Tras la firma de los Acuerdos de Paz, Ralph creía que la posibilidad de un cambio estructural era posible, sin embargo, al poco tiempo se desencantó. Esto lo llevó a tomar distancia, lo cual no se transformó exactamente en desinterés, sino en una continua reflexión y búsqueda de respuestas.

En los Países Bajos, Ralph decidió retomar sus estudios académicos y siguió una maestría en la Universidad de Ámsterdam, en el Centro de Estudios y Documentación para América Latina (CEDLA). Disfrutó de aprender y de ser estudiante, y ante este nuevo reto se destacó: en 2004 obtuvo Cum Laude con su investigación The Price of Peace: The Human Right Movement in PostwarEl Salvador. ${ }^{19}$ Mientras estudiaba, Ralph trabajaba para la Organización Intereclesiástica para la Cooperación para el Desarrollo (ICCO), desde donde seguía vinculado a la región centroamericana. En 2009, después de algunos años de haber terminado su maestría, inició su investigación de doctorado con la colaboración de IS Academia de Seguridad Humana en Estados Frágiles, ICCO y la

18 Arzobispado de Guatemala. Oficina de Derechos Humanos, Hasta Encontrarte: Niñez desaparecida por el conflicto armado interno en Guatemala (Guatemala: Oficina de Derechos Humanos del Arzobispado de Guatemala/Fundación SKIP, 2000), 29.

19 Ralph Sprenkels, The Price of Peace: The Human Right Movement in Postwar El Salvador (Amsterdam, Países Bajos: Center for Latin American Studies and Documentation, CEDLA, 2005). 
Universidad de Utrecht. Siempre interesado en el conflicto y la violencia, eligió nuevamente El Salvador como país para su proyecto de investigación, esta vez se centraría en la posguerra y los veteranos del FMLN. Su trabajo de doctorado se tituló Revolution \& Accommodation Post-Insurgency in El Salvador, ${ }^{20}$ de donde nace posteriormente After Insurgency. Revolution and Electoral Politics in El Salvador, ${ }^{21}$ publicado por la Universidad de Notre Dame.

\section{Su obra académica: Un eterno retorno a EI Salvador}

En el 2001, Ralph decía sobre sus estudios: «Prefiero la vida a la academia, aunque sí me gustan mucho los libros... ».22 Es claro que, en aquel momento, Ralph no podía apreciar la importancia que tendría su experiencia vital para el relevante proyecto de investigación académica que iniciaría unos años después.

Quizá, una manera de comenzar poniendo en perspectiva su producción académica sea traer a colación el recuento realizado por Alberto Martín Álvarez (2014) ${ }^{23}$ sobre los temas abordados en la literatura académica sobre el conflicto armado en El Salvador. Según Martín Álvarez, la década de los ochenta estuvo dominada por la literatura producida por simpatizantes con el movimiento revolucionario o con la lucha del pueblo salvadoreño. Las reflexiones predominantes se relacionan con lo militar y con la teología de la liberación. En la década de los noventa, predominaron las «indagaciones sobre el rol de la ideología y la voluntad insurgente en la guerrilla» ${ }^{24}$ y sobre las características del régimen militar. Finalmente, la producción de la década del dos mil fue más limitada, pero con multiplicación de nuevos intereses de investigación.

En un artículo posterior al referido, en el que se asociaron el mismo Martín Álvarez y Ralph Sprenkels (2014), ${ }^{25}$ esta misma taxonomía fue profundizada. Nos interesa retomar la idea de que los años noventa permitieron la apertura «de una producción con propósitos más académicos que ideológico-políticos [...] de un mayor valor cualitativo». ${ }^{26}$ Además, en el artículo sostienen que la paz y los

20 Véase nota 2.

21 Ralph Sprenkels, After Insurgency. Revolution and Electoral Politics in El Salvador (Notre Dame University, 2018).

22 Ralph Sprenkels, entrevistado por Daniel Eb Huezo Lagos, El Faro, 2001, http://archivo.elfaro.net/ anteriores/2001/111201/secciones/agora/rs.html.

23 Alberto Martín Álvarez, «Estado de la cuestión sobre el conflicto armado. Sus principales tendencias», en: Historia y debates sobre el conflicto armado salvadoreño y sus secuelas, editado por Jorge Juárez (San Salvador, El Salvador: Unidad de Investigación sobre la Guerra Civil Salvadoreña del Instituto de Estudios Históricos, Antropológicos y Arqueológicos, Universidad de El Salvador/Fundación Friedrich Ebert, 2014), 221-223.

24 Ibíd., 222.

25 Alberto Martín Álvarez y Ralph Sprenkels, «La izquierda revolucionaria salvadoreña. Balance historiográfico y perspectivas de investigación», en: El estudio de las luchas Revolucionarias en América Latina (1959-1996), editado por Verónica Oikión Solano, Eduardo Rey Tristán y Martín López Ávalos (México-Santiago de Compostela: El Colegio de Michoacán y Universidad de Santiago de Compostela, 2014).

26 Ibíd., 222. 
tiempos que se abrieron a partir del 2000 aportaron una distancia suficiente para comenzar a pensar nuevos balances de lo ocurrido durante la guerra, entre estos, «las posibles herencias del proceso de lucha armada y guerra revolucionaria». ${ }^{27}$ Estos dos aspectos: distancia crítica de lo ideológico y profundidad académica, por un lado, y balance de las herencias del proceso de lucha armada y guerra revolucionaria, por el otro, son elementos fundantes de la obra de Ralph. En efecto, en primer lugar, Ralph se preocupó, a cada paso, de explicitar la necesidad de tomar distancia de las versiones maniqueas sobre la guerra salvadoreña. En segundo, buena parte de su obra académica buscó vincular cada presente desde el que él escribía, en la posguerra, con los procesos políticos de la guerra.

Debemos señalar que buena parte de su obra sobre El Salvador está fuertemente marcada por varios elementos que se podrían dividir en dos grandes aspectos. Primero, los de carácter histórico, vinculados al momento de su llegada al país, esto es: la transición política producto de los acuerdos de paz, la Ley de Amnistía -que impidió que los crímenes de lesa humanidad fueran juzgados-, así como la continuidad de la polarización política, esta vez en el contexto de la naciente democracia. Como veremos, el estudio de la posguerra en El Salvador es medular en su obra, aunque posteriormente se irá acercando, cada vez más, al estudio del período de la guerra y sus antecedentes. El segundo aspecto tiene que ver con lo más íntimo, con su militancia por los derechos humanos a la que nos hemos referido.

Así pues, entre 2001 y 2004, las primeras obras de Ralph estuvieron dedicadas, precisamente, a los niños y a las niñas desaparecidos. Tal es el caso de los libros El día más esperado. Buscando a los niños desaparecidos de El Salvador, de la Asociación Pro-Búsqueda, publicado en 2001 e Historias para tener presente, del 2002. ${ }^{28}$ Este último tiene una traducción al inglés del 2015. ${ }^{29} \mathrm{El}$ día más esperado busca reconstruir «la historia y los sucesos de la guerra por los cuales desaparecieron los niños y niñas en El Salvador. Los familiares relatan la génesis de Pro-Búsqueda y los esfuerzos realizados en su localización $[\ldots] »,{ }^{30}$ así como la problemática de estos niños y las implicaciones políticas y sociales.

En el segundo libro, Ralph explicita el papel que desempeñó: «mi papel de facilitador del proyecto y editor del libro se centró en ayudar a los jóvenes a vencer los obstáculos prácticos que aparecieron al comenzar la redacción de los relatos». ${ }^{31} \mathrm{Su}$ labor, en esta línea, tuvo impacto en Guatemala, otro de los países centroamericanos en el que se negoció una salida al conflicto armado. Ralph

27 Ibíd., 225.

28 Sprenkels, El día más esperado...; Ralph Sprenkels, ed., Historias para tener presente (San Salvador, El Salvador: Asociación Pro-Búsqueda de Niñas y Niños Desaparecidos y UCA Editores, 2002).

29 Ralph Sprenkels, ed., Stories never to be forgotten. Eyewitness accounts from the Salvadorian civil war (Arizona, EE. UU.: Bilingual Press, 2015).

30 Sprenkels, El día más esperado..., 19.

31 Sprenkels, Stories never to be forgotten..., xii. 
acompañó a la arquidiócesis de este país en el primer estudio exploratorio sobre la niñez desaparecida, cuya primera versión fue publicada en el 2000. ${ }^{32}$

En esta época, se comienza a definir un elemento que atravesará, teóricamente, gran parte de la obra de Ralph en los años por venir, y es el concepto de la memoria. En estos primeros años de la década del 2000, y en el marco de Pro-Búsqueda, él señalaba que «hablar de la memoria es importante, porque su lucha es precisamente la recuperación de la memoria para que pueda aparecer la verdad».33

Al posterior tratamiento de la memoria que realizó Ralph, nos referiremos más adelante. Antes quisiéramos dar seguimiento cronológico a su trabajo intelectual. En este sentido, en The Price of Peace. The Human Rights Movement in Postwar El Salvador, del 2005, se detecta un giro en el trabajo intelectual de Ralph. Publicado en los Cuadernos del Centre for Latin American Studies and Documentation, Ámsterdam, esta obra es, seguramente, un trabajo de graduación universitaria. The Price of Pace muestra la confluencia de su trabajo de diez años en el campo de los derechos humanos en El Salvador con su labor de corte académico. Esto será, a partir de este momento, una práctica recurrente. En esta obra, Ralph se pregunta: «¿Cómo afectó la transición democrática de la posguerra al movimiento de derechos humanos de El Salvador? ¿Cuáles fueron los procesos -sociales- subyacentes que determinaron este desarrollo?». ${ }^{34}$ Analizando varias organizaciones de familiares de desaparecidos, Ralph abarca el período que transcurre entre la década de 1970 y el final de la década de 1990. Ralph muestra que el surgimiento de los movimientos pro derechos humanos, en la década de los setenta, tuvo como uno de sus puntos de agenda el debilitamiento o la derrota del régimen político salvadoreño, por lo que su alianza con el Frente Farabundo Martí para la Liberación Nacional (FMLN) era motivo de múltiples acusaciones. A mediados de la década de 1980, en medio de tales acusaciones, el movimiento pro derechos humanos buscó ganar legitimidad a través de la definición de su identidad como familiares de asesinados y desaparecidos y, por lo tanto, como familiares agraviados. Con los acuerdos de paz y la aprobación de una Ley de Amnistía que amparó a su aliado izquierdista por los crímenes cometidos durante la guerra civil, afloraron las contradicciones entre el FMLN y el movimiento pro derechos humanos, debilitando a estos últimos. No nos detendremos en los elementos conceptuales de este trabajo en particular, pero aquí es ya observable el gusto intelectual de Ralph por el sociólogo norteamericano

32 Arzobispado de Guatemala. Oficina de Derechos Humanos. Hasta Encontrarte... También puede encontrarse una edición posterior: Arquidiócesis de Guatemala. Hasta encontrarte: Niñez desaparecida por el conflicto armado interno en Guatemala (Guatemala: Oficina de Derechos Humanos del Arzobispado de Guatemala/Fundación SKIP, 2002).

33 Ralph Sprenkels, «Pro-Búsqueda. Heridas pasadas, heridas presentes. Actualidad de la experiencia de la guerra en El Salvador, analizada desde la búsqueda de niñas y niños desaparecidos», en: Educar desde la memoria, ed. por Asociación de Mujeres por la Dignidad y la Vida «Las Dignas» (San Salvador, El Salvador: Asociación de Mujeres por la Dignidad y la Vida «Las Dignas», 2004), 37-55, 38.

34 Sprenkels, The Price of Peace..., 5. 
Charles Tilly. A este autor recurrirá, muy frecuentemente, en los trabajos venideros. A partir de aquí también se hará presente su interés en la posguerra salvadoreña, siempre en contrapunto con el pasado, así como en la investigación de la importancia del izquierdista FMLN en el nuevo escenario político que, sin embargo, siguió marcado por la polarización. Ralph también comenzará a preguntarse por la identidad vinculada a la memoria.

La polarización política marcada por el FMLN y el derechista Partido Alianza Republicana Nacionalista (ARENA) fue el objeto de análisis de Ralph desde la perspectiva de la memoria. A partir de este momento, en la obra de Ralph, el concepto de «memoria» dejó de estar delineado por la idea del trabajo de «desenterrar la verdad», ${ }^{35} \mathrm{y}$ su investigación fue cobrando mayor complejidad conceptual y analítica, valga decir, una mayor sofisticación.

Es el período que va de 2011 a 2017 en el que la obra de Ralph estuvo especialmente marcada por esta tendencia analítica. Sobre esta, nos referiremos a continuación. En el 2011 aparecieron publicados dos trabajos dedicados al análisis de distintas dimensiones de la memoria de dos figuras políticas centrales para El Salvador: Roberto d'Abuisson, principal líder de la derecha vinculado a los escuadrones de la muerte y Schafik Hándal del partido de izquierda FMLN y antiguo miembro del Partido Comunista. ${ }^{36}$

En el caso de Roberto d'Aubuisson, Ralph muestra cómo el trabajo de la memoria tuvo el fin de rehabilitarlo de su calidad de líder de los escuadrones de la muerte y de partícipe del asesinato de Monseñor Óscar Arnulfo Romero. Sobre él se construyó una memoria de líder señero, cristiano excepcional, «un héroe que salva a El Salvador de la amenaza comunista». ${ }^{37}$ Por otra parte, Schafik Hándal fue edificado como un «gigante de la Historia», el artífice de los acuerdos de paz que abrieron el camino a la democracia en El Salvador, así como al triunfo electoral del $2009 .{ }^{38}$ Ralph concluye que:

Las similitudes del trabajo de la memoria política en relación con Roberto d'Aubuisson y Schafik Handal son obvias: los partidarios los proyectan como ciudadanos extraordinarios, o de impecables estándares morales, visionarios y amantes de la libertad, víctimas de persecución y calumnia, oradores dotados, cercanos a la gente y amados por esta, dispuestos a soportar cualquier sacrificio imaginado por sus ideales. Ambos han sido construidos como «padres fundadores» de la nación

35 Sprenkels, «Pro-Búsqueda. Heridas pasadas, heridas presentes...», 39.

36 Sprenkels, Ralph, «Roberto d'Aubuisson vs Schafik Handal: Militancy, Memory Work and Human Rights» European. Review of Latin American and Caribbean Studies, n. ${ }^{\circ} 91$ (2011): 15-30, https://www. jstor.org/stable/41305291; Sprenkels, Ralph, «La memoria militante. Historia y política en la posguerra salvadoreña», en: Conflicto, memoria y pasados traumáticos: El Salvador contemporáneo, coordinado por Eduardo Rey y Pilar Cagiao (Santiago de Compostela, España: Universidad de Santiago de Compostela, 2011).

37 Sprenkels, «Roberto d'Aubuisson vs Schafik Handal...», 20.

38 Ibíd. 
salvadoreña. Mientras tanto, el adversario político destaca como fuerza perversa que debe de ser vencida. ${ }^{39}$

En el contexto de estas investigaciones, Ralph elabora el concepto de «memorias militantes», para lo cual recurre, por una parte, a la categoría de «marcos» desarrollada por Goffman, la cual «puede ser conceptualizada como «paquetes interpretativos» que los activistas desarrollan para movilizar potenciales partidarios y votantes, atraer a autoridades y desmovilizar adversarios $» .{ }^{40}$ Por otra parte, utiliza el concepto elaborado por Charles Tilly de «historias modelos», entendido como «narrativas explicativas que incorporan un limitado número de actores, precisamente solo en unas pocas acciones, y simplifican las interpretaciones de causa y efecto en las cuales las acciones de los actores producen todo el resultado significativo».$^{41}$ Ralph desarrolló un muy sofisticado aparataje conceptual a partir de estas categorías, junto con la de memoria colectiva, que no es un recuerdo sino una declaración de lo ocurrido, una práctica política -en el sentido de Maurice Halbwachs-, «un producto de procesos sociales de construcción e interpretación de sucesos históricos, una generadora de identidades». ${ }^{42}$

Luego de estos trabajos, Ralph siguió explorando la maleabilidad del pasado a partir de su uso político, en una posguerra aún polarizada por las antiguas fuerzas en disputa. Esto lo hizo en dos investigaciones: una, a propósito de la violencia que generaron las protestas el 5 julio de $2006,{ }^{43}$ otra, a partir del uso político del Informe de la Comisión de la Verdad para el Salvador. ${ }^{44}$

Sobre el primer trabajo mencionado debe decirse que el 5 de julio del 2006 un grupo de jóvenes protestó en San Salvador contra el alza de las tarifas al transporte público, lo cual desembocó en violencia armada entre manifestantes y fuerzas del orden. A Ralph le interesaba destacar lo que las interpretaciones y manipulaciones realizadas desde la izquierda y la derecha partidarias «pueden enseñarnos sobre la dinámica política de la posguerra salvadoreña». ${ }^{45}$ Para ello Ralph analizó, por una parte, el discurso anticomunista histórico de la derecha salvadoreña, y por otra, el de la izquierda revolucionaria transformada en partido electoral. Ralph señala:

Los derechistas explicaron los disturbios como un hecho de terrorismo de izquierda inscrito en un complot más grande para desestabilizar un gobierno legítimo,

39 Ibíd., 24.

40 Ibíd., 17

41 Ibíd.

42 Sprenkels, «La memoria militante...», 269.

43 Ralph Sprenkels, «Arena, FMLN y los sucesos del 5 de julio del 2006 en El Salvador: violencia e imaginarios políticos», TRACE, n. ${ }^{\circ} 66$ (2014): 62-81, https://www.redalyc.org/articulo.oa?id=423839524004.

44 Ralph Sprenkels, «La guerra como controversia: una reflexión sobre las secuelas políticas del informe de la Comisión de la Verdad para El Salvador», Identidades, año 2, n. ${ }^{\circ} 4$ (2012): 68-89, https://oibc.oei.es/ uploads/attachments/212/identidades-3.pdf.

45 Sprenkels, «Arena, FMLN y los sucesos del 5 de julio...», 66. 
mientras que los de izquierda interpretaron los hechos como una conspiración de la derecha para desacreditar al FMLN y de esa manera justificar la represión en contra del creciente descontento popular. Construyendo estas interpretaciones, tanto una como otra se remitió a la participación del contrincante en hechos de violencia política antes y durante la guerra. Ambos bandos echaron mano de la representación del enemigo forjada en el conflicto armado. ${ }^{46}$

En el segundo trabajo referido, Ralph analizó la controversia en torno al Informe de la Comisión de la Verdad, en el que se responsabilizó a las fuerzas de derecha e izquierda por algunos de los crímenes de guerra cometidos. La controversia la entiende Ralph como «la manera en que actores políticos buscan "darle vuelta" a hechos, datos e interpretaciones, replicando acusaciones de rivales políticos y desviando posibles costos políticos»». ${ }^{47}$

La estrategia política de la derecha, aglutinada en el partido ARENA, consistió en enfocar el Informe «como un requisito cumplido para poder proceder con una política de "borrón y cuenta nueva", basada en la doble acción de perdonar y olvidar los crímenes de guerra». ${ }^{48}$ Para la izquierda, la estrategia consistió en eludir la derogación de la Ley de Amnistía que evitó el juzgamiento de los crímenes señalados en el Informe, entrando en tensiones con los movimientos pro derechos humanos. Así, según Ralph, «después de la Comisión de la Verdad, los militantes salvadoreños de derecha y de izquierda se siguieron tomando la licencia de reescribir la historia a su medida, a modo de lograr una síntesis casi perfecta de la historia con sus intereses políticos actuales»». ${ }^{49}$

Un trabajo que recoge los rasgos sustantivos de su investigación sobre la memoria fue publicado en Costa Rica en el 2017. ${ }^{50}$ En él, apoyado en otros autores, buscó dar cuenta de cinco propuestas heurísticas en torno a las guerras en El Salvador, Guatemala y Nicaragua: la del olvido impuesto, la del desengaño respecto de los dirigentes, la fraternicida -que ve a las diferentes partes como hermanos de un mismo pueblo-, la heurística revolucionaria y la anticomunista. A partir de un análisis comparativo entre los procesos conflictivos de los tres países, arrojó luces sobre similitudes y particularidades de cada caso. Sin duda, un trabajo de referencia para quien busque analizar las memorias en Centroamérica.

Para finalizar este apartado, quisiéramos señalar tres aspectos de la obra de Ralph. El primero es que su análisis de la posguerra encontró su máxima expresión en el libro After Insurgency: Revolution and Electoral Politics in El Salvador, publicado en 2018, producto de su tesis doctoral. Esta es una obra

46 Ibíd., 68.

47 Sprenkels, «La guerra como controversia...», 68.

48 Ibíd., 73.

49 Ibíd., 85.

50 Ralph Sprenkels, «El trabajo de la memoria en Centroamérica: Cinco propuestas heurísticas en torno a las guerras en El Salvador, Guatemala y Nicaragua», Revista de Historia, n. ${ }^{\circ} 76$ (julio-diciembre, 2017): 1346, http://dx.doi.org/10.15359/rh.76.1. 
dedicada al análisis de la reconversión del FMLN en su paso de guerrilla a un exitoso partido político. ${ }^{51}$ En esta misma línea publicó un artículo a inicios del 2019, una de cuyas principales conclusiones sobre la evolución política de la antigua izquierda es:

\begin{abstract}
El acomodo ideológico del FMLN en la posguerra se entiende mejor como moderación ambivalente. Si bien la moderación ideológica proporcionó recompensas electorales, la continuidad de la ideología revolucionaria fue más efectiva para resolver disputas entre las fracciones y la consolidación del liderazgo. ${ }^{52}$
\end{abstract}

El segundo aspecto alude al trabajo de investigación realizado por Ralph sobre justicia transicional, área en la que posee al menos dos obras. ${ }^{53} \mathrm{Sin}$ embargo, ya en diciembre de 2014, Ralph había realizado una discusión sobre desarme, desmovilización y reintegración, para lo cual reconstruyó las trayectorias de vida de excombatientes del FMLN a partir de fotografías históricas. ${ }^{54} \mathrm{En}$ esta investigación, señaló que el concepto de «reconversión» es más adecuado que el concepto de «reintegración» para captar la dinámica mediante la cual se adaptan los grupos insurgentes en el proceso de transitar de la guerra a la paz. Esto, debido al uso de distintas formas de capital que acumularon los involucrados durante la guerra en el nuevo proceso de la posguerra.

El tercer y último aspecto que quisiéramos retomar tiene que ver con la apertura paulatina que hizo Ralph hacia la investigación sobre el período de la guerra civil salvadoreña propiamente dicha, ya no en función de la comprensión de la posguerra. En asocio con Martín Álvarez, ya había analizado en el 2014 la producción sobre la izquierda revolucionaria. ${ }^{55}$ Ese mismo año, Ralph publicó un análisis de las relaciones urbano-rurales en la insurgencia salvadoreña. ${ }^{56}$ Además, en el libro La revolución revisitada, publicado en el 2017, incluyó un artículo, elaborado junto a Michelle Melara Minero, sobre los patrones, variaciones y actores de la persecución violenta en el período 1970-1991. ${ }^{57}$

51 Sprenkels, After Insurgency: Revolution and ...

52 Ralph Sprenkels, «Ambivalent Moderation: The FMLN's Ideological Accommodation to Post-War Politics in El Salvador». Government and Opposition, vol. 54, n. ${ }^{\circ} 3$ (2019): 536-558, 251, http://dx.doi. org/10.1017/gov.2018.37.

53 Ralph Sprenkels, Restricted Access' Promises and Pitfalls of Victim Participation in Transitional Justice Mechanisms A Comparative Perspective (Utrecht, Países Bajos: Impunity Watch, 2017); Leticia Orduña, Ralph Sprenkels y Jorge Juárez, coords., La justicia transicional en perspectiva comparada: Centroamérica y México (México: Centro de Investigación sobre América Latina y El Caribe-UNAM, 2018).

54 Ralph Sprenkels, Reintegration or reconversion? Probing the nature of Disarmament, Demobilisation and Reintegration (DDR) processes in fragile settings (The Netherlands: IS Academy on Human Security in Fragile States, 2014).

55 Alberto Martín Álvarez y Ralph Sprenkels, «La izquierda revolucionaria salvadoreña...»

56 Ralph Sprenkels, «Las relaciones urbano-rurales en la insurgencia salvadoreña», en: Historia y debates sobre el conflicto armado salvadoreño y sus secuelas, coordinado por Jorge Alberto Juárez Ávila (San Salvador, El Salvador: Instituto de Estudios Históricos, Antropológicos y Arqueológicos, 2014), 25-43.

57 Sprenkels, Ralph y Melara Minero, Lidice M., «Auge y declive de la persecución violenta en El Salvador: Patrones, variaciones y actores (1970-1991)», en: La revolución revisitada. Nuevas perspectivas sobre la 


\section{Reconstruyendo memorias y demandando justicia: EI Mozote}

El 2018 fue un año significativo que implicó la condensación de dos de las vertientes que Ralph cultivó de manera tan sólida y comprometida: el trabajo académico y aquel en pro de los derechos humanos en El Salvador. Efectivamente, ese año Ralph fue designado como especialista académico en el peritaje ${ }^{58}$ de la masacre de El Mozote, acontecida en el norte de Morazán en 1981, y donde fueron asesinadas más de mil personas en un operativo militar ejecutado por el Batallón de Infantería de Reacción Inmediata Atlactatl. La Ley de Amnistía aprobada en 1993, días después del Informe de la Comisión de la Verdad, había cerrado la posibilidad de investigación judicial sobre la responsabilidad de estos hechos. Sin embargo, en el 2016, la Sala Constitucional de la Corte Suprema de Justicia anuló la ley aprobada en 1993, y el juez del Juzgado Segundo de Primera Instancia de San Francisco Gotera abrió el caso. ${ }^{59}$ La responsabilidad y el impacto de este proceso eran significativos para Ralph. El Mozote representaba el caso emblemático que estaba abriendo la posibilidad de establecer reconocimiento, justicia y reparación a las víctimas y sus familiares en El Salvador.

\section{No hay conclusión: «Mientras dure la vida sigamos con el cuento» ${ }^{60}$}

En agosto de 2019, Ralph iba a comenzar su nuevo trabajo en la Universidad de Leiden como profesor de Historia Latinoamericana. Estaba entusiasmado, en su propuesta de agenda llevaba varios proyectos que desde hacía años quería realizar, El Salvador seguía siendo el punto medular de su interés académico. Estaba convencido de que aún quedaba mucho camino por recorrer en la investigación sobre el conflicto armado y la posguerra, entre los casos que él consideraba como pendientes destacaba el complejo rompecabezas del conflicto interno dentro de las Fuerzas Populares de Liberación (FPL) que decantó en el asesinato de la comandante Mélida Anaya Montes, conocida como Ana María, y que supuestamente fue ejecutado bajo las órdenes del comandante Salvador Cayetano Carpio, conocido como Marcial. Según investigaciones, Marcial se suicidó días después de la muerte de Ana María. Ralph consideraba que este episodio no estaba cerrado, había muchos cabos sueltos en la investigación, y le interesaba cómo en la posguerra la figura de Marcial seguía siendo un referente para muchos veteranos de guerra, a pesar de su controversial figura.

insurrección y la guerra en El Salvador, editado por Mauricio Menjívar y Ralph Sprenkels (San Salvador, El Salvador: UCA Editores, 2017), 79-148.

58 Más información sobre el caso, véase: https://www.laprensagrafica.com/elsalvador/Juramentan-a-peritos-que-investigaran-el-impacto-social-de-macracre-El-Mozote-20190212-0221.html y https:/www.lapagina.com.sv/nacionales/juramentan-dos-nuevos-peritos-en-caso-el-mozote/.

59 Fátima Peña, «Juez ordena reapertura del caso El Mozote y abre proceso contra el Alto Mando de 1981», El Faro Digital, 30 de septiembre de 2016. https://elfaro.net/es/201609/el_salvador/19339/Juez-ordena-reapertura-del-caso-El-Mozote-y-abre-proceso-contra-el-Alto-Mando-de-1981.htm.

60 Frase utilizada por Ralph Sprenkels en su cumpleaños número 50. 
Ralph era un académico pujante, activo, reflexivo y en continua búsqueda. En su obra existen elementos teóricos, metodológicos y líneas de investigación abiertas a las nuevas generaciones de investigadoras e investigadores de la realidad salvadoreña. También fue un militante por los derechos humanos, cuya labor tuvo impacto sensible sobre la vida de quienes sobrevivieron al horror de la barbarie. Ambas vertientes son parte de su legado, y una de las razones por las que personas de diferentes orígenes y nacionalidades lo evocarán en nuevos presentes, con aprecio y admiración. Ralph es ya, de manera ineludible, un referente de la historia intelectual y de la lucha por la justicia en el Pulgarcito de América. No hay duda, Ralph es una inspiración para que «sigamos con el cuento».

\section{Bibliografía}

Arzobispado de Guatemala. Oficina de Derechos Humanos. Hasta Encontrarte: Niñez desaparecida por el conflicto armado interno en Guatemala. Guatemala: Oficina de Derechos Humanos del Arzobispado de Guatemala/ Fundación SKIP, 2000.

Arquidiócesis de Guatemala. Hasta encontrarte: Niñez desaparecida por el conflicto armado interno en Guatemala. Guatemala: Oficina de Derechos Humanos del Arzobispado de Guatemala/Fundación SKIP, 2006.

Martín Álvarez, Alberto. «Estado de la cuestión sobre el conflicto armado. Sus principales tendencias». En: Historia y debates sobre el conflicto armado salvadoreño y sus secuelas, editado por Jorge Alberto Juárez Ávila. San Salvador, El Salvador: Unidad de Investigación sobre la Guerra Civil Salvadoreña del Instituto de Estudios Históricos, Antropológicos y Arqueológicos. Universidad de El Salvador/Fundación Friedrich Ebert, 2014.

Martín Álvarez, Alberto y Sprenkels, Ralph. «La izquierda revolucionaria salvadoreña. Balance historiográfico y perspectivas de investigación». En: El estudio de las luchas revolucionarias en América Latina (1959-1996), editado por Verónica Oikión Solano, Eduardo Rey Tristán y Martín López Ávalos. México-Santiago de Compostela: El Colegio de Michoacán y Universidad de Santiago de Compostela, 2014.

Sprenkels, Ralph (ed.). El día más esperado. Buscando a los niños desaparecidos de El Salvador. San Salvador, El Salvador: Asociación Pro-Búsqueda de niñas y niños desaparecidos/UCA Editores, 2001.

Ralph Sprenkels. «El día más esperado». Entrevista por Daniel Eb Huezo Lagos. El Faro, (2001). http://archivo.elfaro.net/anteriores/2001/111201/ secciones/agora/rs.html.

Sprenkels, Ralph (ed.). Historias para tener presente. San Salvador, El Salvador: Asociación Pro-Búsqueda de Niñas y Niños Desaparecidos y UCA Editores, 2002. 
Sprenkels, Ralph «Pro-Búsqueda. Heridas pasadas, heridas presentes. Actualidad de la experiencia de la guerra en El Salvador, analizada desde la búsqueda de niñas y niños desaparecidos». En: Educar desde la memoria, editado por Asociación de Mujeres por la Dignidad y la Vida «Las Dignas». San Salvador, El Salvador: Asociación de Mujeres por la Dignidad y la Vida «Las Dignas», 2004.

Sprenkels, Ralph. The Price of Peace. The Human Rights Movement in Postwar El Salvador. Amsterdam, Países Bajos: Center for Latin American Studies and Documentation, 2005.

Sprenkels, Ralph. «Een messcherpe scheidslijn. Chalatenango, El Salvador: Toen en nu». En: Overleven Verplicht. 25 jaar na de moord op de Nederlandse jounalisten in El Salvador, uitgegeven door Marc Broere. Amsterdam, Países Bajos: OnzeWereld Media, 2007.

Sprenkels, Ralph. Caminar con el pueblo. Entrevista con Jon cortina. San Salvador, El Salvador: Ediciones Populares, 2009.

Sprenkels, Ralph. «Roberto d'Aubuisson vs Schafik Handal: Militancy, Memory Work and Human Rights» European. Review of Latin American and Caribbean Studies, n. ${ }^{\circ} 91$ (2011), 15-30. https://www.jstor.org/stable/41305291.

Sprenkels, Ralph. «La memoria militante. Historia y política en la posguerra salvadoreña». En: Conflicto, memoria y pasados traumáticos: El Salvador contemporáneo, coordinado por Eduardo Rey y Pilar Cagiao. Santiago de Compostela, España: Universidad de Santiago de Compostela, 2011.

Sprenkels, Ralph. «La guerra como controversia: Una reflexión sobre las secuelas políticas del informe de la Comisión de la Verdad para El Salvador». Identidades, año 2, n. ${ }^{\circ} 4$ (2012): 68-89. https://oibc.oei.es/uploads/attachments/212/identidades-3.pdf.

Sprenkels, Ralph. «Las relaciones urbano-rurales en la insurgencia salvadoreña». En: Historia y debates sobre el conflicto armado salvadoreño y sus secuelas, coordinado por Jorge Alberto Juárez Ávila. San Salvador, El Salvador: Instituto de Estudios Históricos, Antropológicos y Arqueológicos, 2014.

Sprenkels, Ralph. Reintegration or reconversion? Probing the nature of Disarmament, Demobilisation and Reintegration (DDR) processes in fragile settings. The Netherlands: IS Academy on Human Security in Fragile States, 2014a.

Sprenkels, Ralph. «Revolution and Accommodation. Post-Insurgency in El Salvador». Tesis de Doctorado, Universidad de Utrecht, Países Bajos, 2014b.

Sprenkels, Ralph. «Arena, FMLN y los sucesos del 5 de julio del 2006 en El Salvador: Violencia e imaginarios políticos», TRACE n. ${ }^{\circ} 66$ (2014): 62-81. https://www.redalyc.org/articulo.oa?id=423839524004. 
Sprenkels, Ralph (ed.). Stories never to be forgotten. Eyewitnes accounts from the Salvadorian civil war. Arizona, EE. UU.: Bilingual Press, 2015.

Sprenkels, Ralph. «El trabajo de la memoria en Centroamérica: Cinco propuestas heurísticas en torno a las guerras en El Salvador, Guatemala y Nicaragua». Revista de Historia, n. ${ }^{\circ} 76$ (julio-diciembre, 2017): 13-46. http:// dx.doi.org/10.15359/rh.76.1.

Sprenkels, Ralph. Restricted Access' Promises and Pitfalls of Victim Participation in Transitional Justice Mechanisms A Comparative Perspective. Utrecht, Países Bajos: Impunity Watch, 2017.

Sprenkels, Ralph y Melara Minero, Lidice M. «Auge y declive de la persecución violenta en El Salvador: Patrones, variaciones y actores (1970-1991)». En: La revolución revisitada. Nuevas perspectivas sobre la insurrección y la guerra en El Salvador, editado por Mauricio Menjívar y Ralph Sprenkels. San Salvador, El Salvador: UCA Editores, 2017.

Sprenkels, Ralph. After Insurgency: Revolution and Electoral Politics in El Salvador. Notre Dame: University of Notre Dame Press, 2018.

Sprenkels, Ralph. «Ambivalent Moderation: The FMLN's Ideological Accommodation to Post-War Politics in El Salvador». Government and Opposition, vol. 54, n. ${ }^{\circ} 3$ (2019): 536-558. http://dx.doi.org/10.1017/gov.2018.37.

Orduña, Leticia, Sprenkels, Ralph y Juárez, Jorge (coords.). La justicia transicional en perspectiva comparada: Centroamérica y México. México: Centro de Investigación sobre América Latina y El Caribe-UNAM, 2018. 